\title{
TAUT FOLIATIONS OF SOME SURFACE BUNDLES OVER CIRCLE $\mathbb{S}^{1}$
}

\section{HAMIDOU DATHE and RENOVAT NKUNZIMANA}

Department of Mathematics and Computer Sciences (UCAD)

Dakar

Senegal

e-mail: hamidou.dathe@yahoo.fr nkunzimana_renovat@yahoo.fr

\begin{abstract}
In this article, we give some theorems of classification for codimension one taut foliations on 3-manifolds which are $\sum$-bundles $\pi: V \rightarrow \mathbb{S}^{1}$ over the circle, where $\sum$ is surface of genus $g>1$, and atoroidals with a hyperbolic monodromy $\varphi: \sum \rightarrow \sum$.
\end{abstract}

\section{Introduction}

In [11], Dathe and Tarquini showed that if $\mathcal{F}$ is a codimension 1 foliation without compact leaf on a $\mathbb{T}^{2}$-bundle $\pi: V \rightarrow \mathbb{S}^{1}$ over $\mathbb{S}^{1}$ which is transverse to the fibration, then $\mathcal{F}$ is isotopic to one of the model foliations constructed on that bundle. In this article, we shall show a similar result in the case where the fibers are closed and oriented

2010 Mathematics Subject Classification: 53C12, 57R30, 57R32.

Keywords and phrases: taut foliations, improved general position, pseudo-Anosov, 3-manifold, hyperbolic bundles, contact structures, symplectically fillable.

Received July 2, 2017

(ㄷ) 2017 Scientific Advances Publishers 
surfaces $\sum$ of genus $g>1$. We consider that the foliation is in optimal position with respect to the fibration which is the equivalent notion to transversality in the case where the fibers are tori $\mathbb{T}^{2}$.

We shall also study the effects of small perturbations on that foliation with respect to the fibration. In fact, it's well known that for the hyperbolic 3-manifolds $V$ which $\sum$-bundles over circle $\mathbb{S}^{1}$, the folaition whose leaves are fibers of the fibration is taut. Therefore, it is natural to ask the question whether all taut foliations on $V$ are a result of perturbations of the fibration. In general the answer to this question is negative. We shall show that for the atoroidals 3-manifolds there are taut foliations which are not perturbations of the fibration and we shall give the condtions for which the answer to this question is affirmative.

\section{Hyperbolic Diffeomorphism and Bundles}

In this section, $\sum$ is a closed and oriented surface of genus $g>1$.

Definition 2.1. Let $\varphi: \sum \rightarrow \sum$ be a diffeomorphism and $\varphi_{*}$ : $H_{1}\left(\sum, \mathbb{R}\right) \rightarrow H_{1}\left(\sum, \mathbb{R}\right)$ the isomorphism induced by $\varphi$ in homology. We say that $\varphi$ is hyperbolic if for all $n>0$, the subspace Fix $\left(\varphi_{*}^{n}\right)$ of the fixed points by $(\varphi)_{*}^{n}$ is trivial.

By a singular foliation on $\sum$, we mean a codimension 1 foliation with 4-branche saddl singularities without linking between them. A measured foliation on $\sum$ will be a foliation (eventually singular) equipped with a transverse measurement which is invariant by holonomy and whose completed support is equal to $\sum$.

Let us notice that a measured foliation is transversely oriented if only if it's defined by a closed 1-form [8]. 
Two measured foliations will be said to be transverse if they have the same singular set $K$, are transverse on $\sum-K$ and in the neighbourhood of each point of $K$ we have the following local model:

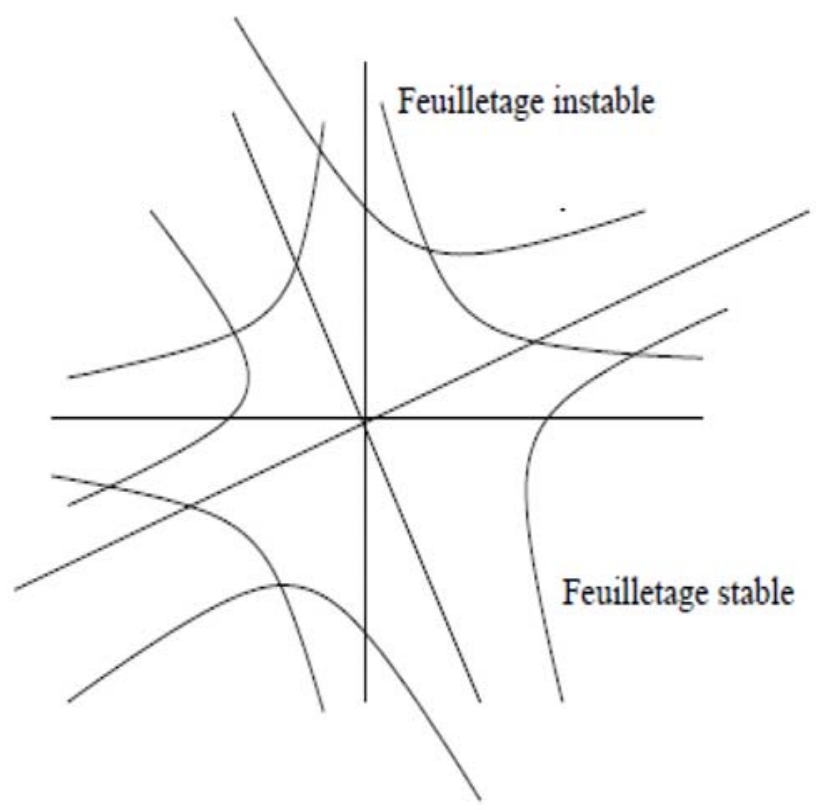

Figure 1. Measured transverse foliations (feuilletage (in)-stable = (un)stable foliation).

Definition 2.2. A diffeomorphism $\varphi$ on $\sum$ is said to be pseudoAnosov if there are two measured transverse foliations $\mathcal{F}^{+}$and $\mathcal{F}^{-}$and a real number $\lambda>1$ satisfying:

$$
\varphi_{*}\left(\mathcal{F}^{+}\right)=\lambda \mathcal{F}^{+} .
$$

$$
\varphi_{*}\left(\mathcal{F}^{-}\right)=\frac{1}{\lambda} \mathcal{F}^{-}
$$

The diffeomorphism $\varphi$ will be said to be periodic if there exists an integer $n>0$ such that $\varphi^{n}=I d$. We say that $\varphi$ is reducible if there 
exists a finite family of simple curves $\left\{\gamma_{1}, \gamma_{2}, \ldots, \gamma_{n}\right\}$ of $\sum$ which are not homotopic to 0 and not homotopic between themselves and are exchanged by $\varphi$.

When the foliations $\mathcal{F}^{+}$and $\mathcal{F}^{-}$are oriented we say that $\varphi$ is an oriented pseudo-Anosov diffeomorphism. In this case the foliations $\mathcal{F}^{+}$ and $\mathcal{F}^{-}$are defined by closed 1-forms. Therefore an oriented pseudoAnosov diffeomorphism is given by a diffeomorphism $\varphi: \sum \rightarrow \sum$, two closed 1 -forms $\omega^{+}, \omega^{-}$and a constant $\lambda>1$ satisfying the following conditions:

(i) $\omega^{+}$and $\omega^{-}$have the same singular set $K$ of isolated points (so in finite number), they are $C^{\infty}$ on $\sum-K$ and in the neighbourhood of each point of $K$, there exists an open set $U$ in which we have $\omega=\omega_{0}=x d x-$ $y d y$.

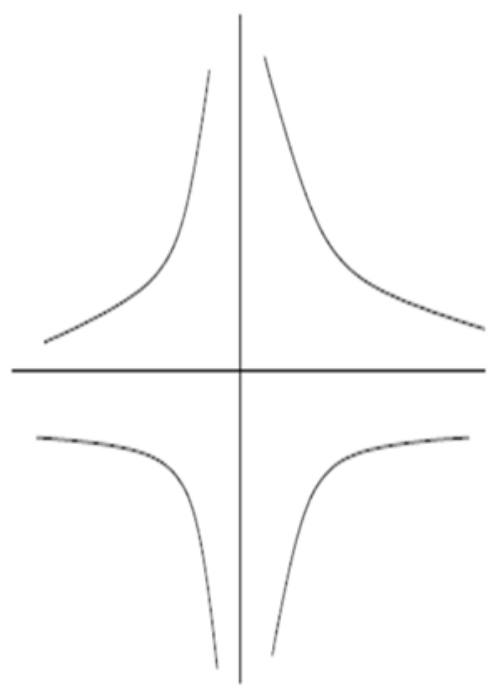

Figure 2. Singularity of saddle type. 
The center-type singularities are locally defined by the 1-form $\omega^{\prime}=x d x+y d y$.

(ii) $\omega^{+}$and $\omega^{-}$are mutually transverse, i.e., $\left(\omega^{+} \wedge \omega^{-}\right)_{u} \neq 0$ for all $u \in \sum-K$.

(iii) $\varphi^{*} \omega^{+}=\frac{1}{\lambda} \omega^{+}$and $\varphi^{*} \omega^{-}=\lambda \omega^{-}$.

The forms $\omega^{+}$and $\omega^{-}$are called characteristic forms of $\varphi$. We have :

Proposition 2.1. The foliations defined by the characteristic 1-forms of oriented pseudo-Anosov diffeomorphism have all their regular leaves dense.

Proof. Let $\mathcal{S}_{\omega^{+}}$and $\mathcal{S}_{\omega^{-}}$be the foliations associated to the characteristic forms of $\varphi$. The foliations $\mathcal{S}_{\omega^{+}}$and $\mathcal{S}_{\omega^{-}}$have no compact leaf because if there were one compact leaf $\gamma$ the latter would not be homotopic to 0 given the type of singularities. As $\sum$ has, up to homotopy, a finite number of compact leaves (see [1]) and is invariant by $\varphi$, there exists $n>0$ such that $\varphi^{n}[\gamma]=[\gamma]$, which is impossible for a pseudo-Anosov diffeomorphism.

On the other hand, there is no connection between the singularities since a connection should be contracted on dilated, which is impossible. Finally, $\mathcal{S}_{\omega^{+}}$and $\mathcal{S}_{\omega^{-}}$have no leaf cycle (i.e., a set homeomorphic to $\mathbb{S}^{1}$ which is the union of singularities and separatrices). As those are measured foliations, from [8], the no compact regular leaves are dense, so $\mathcal{S}_{\omega^{+}}$and $\mathcal{S}_{\omega^{-}}$have all dense regular leaves.

Recall the following theorem:

Theorem 2.1 ([2]). Let $\varphi$ be a diffeomorphism of $\sum$. Then $\varphi$ is isotopic to a diffeomorphism which is periodic or reducible or pseudoAnosov. 
Proposition 2.2. If $\varphi$ is an hyperbolic diffeomorphism of $\sum$, then $\varphi$ is pseudo-Anosov.

Proof. If $\varphi$ is periodic, there exists $n>0$ such that $\varphi^{n}=I d$ and the subspace of points fixed by $\varphi_{*}$ is $H_{1}\left(\sum\right)$, so $\varphi$ is not hyperbolic.

Let us suppose now that $\varphi$ is reducible and let $\left\{\gamma_{1}, \gamma_{2}, \ldots, \gamma_{n}\right\}$ be a finite family of closed simple curves non homotopic to 0 and not mutually homotopic and which are exchanged by $\varphi$. We distinguish two cases:

(1) One of the $\gamma_{i}$, (let us take, for exemple, $\gamma_{1}$ is not separating): there exists $n>0$ such that $\varphi_{*}^{n}\left[\gamma_{1}\right]=\left[\gamma_{1}\right]$ and since, $\left[\gamma_{1}\right] \neq 0, \varphi$ is not hyperbolic. A closed simple curve $\gamma$ on a surface $\sum$ is said to be separating if $\sum-\gamma$ is not connected.

(2) All the $\gamma_{i}$ are separating; let $\sum_{1}, \sum_{2}, \ldots, \sum_{n}$ the connected components of $\sum$ cut along the $\gamma_{i}$. We have $\partial\left(\sum_{i}\right)=\gamma_{i}$ for all $i=1, \ldots, n$. We can suppose that $\varphi^{n-1}\left(\sum_{1}\right) \neq \sum_{1}$ and $\varphi^{n}\left(\sum_{1}\right)=\sum_{1}$ and that $\varphi^{n}$ is not an Anosov diffeomorphism on $\sum_{1}$ which is a compact surface of genus 1. So Fix $\left(\varphi_{*}^{n}\right) \neq 0$, therefore $\varphi$ is not hyperbolic.

When the stable and unstable foliations of a hyperbolic diffeomorphism $\varphi$ are oriented, we say that $\varphi$ is an oriented hyperbolic diffeomorphism.

Proposition 2.3. If $\mathcal{F}$ is an orientable measured foliation on $\sum$ which is invariant by an oriented pseudo-Anosov $\varphi\left(\right.$ i.e., $\left.\varphi_{*}(\mathcal{F})=\mathcal{F}\right)$, then $\mathcal{F}$ is isotopic to one of the foliations defined by the characteristic forms of $\varphi$. 
Proof. Let $\lambda>1$ be the coefficient of dilation of $\varphi$, and $\mathcal{S}_{\omega^{+}}$and $\mathcal{S}_{\omega^{-}}$ be the foliations associated to the characteristic forms of $\varphi$. Let $\omega$ be a 1 -form defining $\mathcal{F}$, if $\omega$ is not proportional to $\omega^{-}$, we know ([2], exposé 5 $=$ talk 5) that $\lambda^{-n} \varphi^{n}(\mathcal{F})$ converges in the space measured foliations to $i\left(\mathcal{F}, \mathcal{S}_{\omega^{-}}\right) \cdot \mathcal{S}_{\omega^{+}}$, where $i\left(\mathcal{F}, \mathcal{S}_{\omega^{-}}\right)$is the number of intersections of $\mathcal{F}$ and $\mathcal{S}_{\omega^{-}}$. This implies that $\mathcal{S}_{\omega^{+}}=0$, which is a contradiction. Therefore $\omega$ is proportional to $\omega^{-}$.

Definition 2.3. Let $V$ be a $\sum$-bundle over $\mathbb{S}^{1}, V$ is said to be hyperbolic of the monodromy $\varphi: \sum \rightarrow \sum$ is a hyperbolic diffeomorphism.

Proposition 2.4. Let $V$ be a hyperbolic $\sum$-bundle over $\mathbb{S}^{1}$, then $b_{1}(V)=b_{2}(V)=1$, where $b_{i}(V)$ is the $i$-th Betti number.

Proof. On the one hand, using duality, we have $b_{1}(V)=b_{2}(V)$. On the other hand $\pi_{1}(V)$ is generated by $\pi_{1}\left(\sum\right)$ and an element $t$ such that $\forall a \in \pi_{1}\left(\sum\right), \varphi_{*} a=t a t^{-1}$. So $H_{1}(V)$ is generated by $t$ and the elements $t_{1}, t_{2}, \ldots, t_{n}$ invariant by $\varphi_{*}\left(\right.$ i.e., $\left.\varphi_{*} t_{i}=t_{i}\right)$. As $V$ is hyperbolic $F i x\left(\varphi_{*}\right)=0$, i.e., $t_{i}=0$ and thus $\operatorname{dim} H_{1}(V)=1$.

\section{Model Foliations on Hyperbolic Bundles}

Let $\mathcal{F}$ be a codimension 1 transversely oriented foliation on a 3-manifold $V$. We say that $\mathcal{F}$ is transversely affine if it is defined by a 1 -form $\alpha$ such that $d \alpha=\alpha \wedge \beta$, where $\beta$ is a 1 -form verifying $d \beta=0$. The foliation $\mathcal{F}$ is said to be taut if it has a closed transverse which intersects all leaves. In particular, if $\mathcal{F}$ has no compact leaf, then it is taut. If $\mathcal{F}$ has a Reeb component, then it is not taut. 
Consider $V$ be a 3-closed hyperbolic manifold bundle over $\mathbb{S}^{1}$. If $\mathcal{F}$ is a codimension 1 transversely oriented foliation on $V$, we call Euler class of $\mathcal{F}$, the usual Euler class of tangent bundle $T \mathcal{F}$ to $\mathcal{F}$ considered as a vector bundle of rank 2 . The Euler class $\chi(T \mathcal{F})$ is an element of $H^{2}(V, \mathbb{R})$, which is isomorphic to $\mathbb{R}$ for the hyperbolic bundles. But the homology class [ $\sum$ ] of each fiber $\sum$ of $V$ is a generator of $H_{2}(V, \mathbb{R})$. Therefore $\chi(T \mathcal{F})$ is determined by its value on $\left[\sum\right]$. This value is called the Euler number of $\mathcal{F}$ and in the following we will note it by $e(\mathcal{F})$.

\section{Computation of $e(\mathcal{F})$ for a taut foliation $\mathcal{F}$}

Suppose that $\mathcal{F}$ is codimension 1 taut foliation on a closed hyperbolic 3-manifold $V$ bundle over $\mathbb{S}^{1}$. Let $\sum$ be a fiber of $V$; it's well known ([17]) that $\sum$ is isotopic to a closed surface which we denote again $\sum$ and is transverse to $\mathcal{F}$ without in a finite number of points which are saddle singularities with 4 separatrix for $\mathcal{F}$ (we say that $\sum$ is in optimal position $\mathcal{F}$ ). A singularity $s$ is said to be positive (resp., negative) if the orientation of the tangent bundle $T \mathcal{F}$ to $\mathcal{F}$ at $\mathrm{s}$ is the same (resp., opposite) as that of the tangent bundle to the fibration at $s$. Let us denote $I_{p}$ the sum of indices of the positive singularities and $I_{n}$ the sum of indices of the negative singularities. According to ([20]), we have:

$$
I_{p}-I_{n}=e(T \mathcal{F})(1) \text { and } I_{p}+I_{n}=\chi\left(\sum\right)(2) \text {, where } \chi\left(\sum\right) \text { is the }
$$
characteristic of Euler-Poincaré of $\sum$.

The continuation of this article is the study of taut foliations on a closed hyperbolic 3-manifold bundle over circle $\mathbb{S}^{1}$ and hyperbolic. Consider firstly the case where the fiber is torus $\mathbb{T}^{2}$. In this case, the monodromy $\varphi$ is an Anosov diffeomorphism of torus $\mathbb{T}^{2}$ induced by a 
hyperbolic matrix $A$ of $\mathbb{R}^{2}$. The matrix $A$ has two real eigenvalues $\lambda$ and $1 / \lambda$. The foliations of $\mathbb{R}^{2}$ by lines of slope $\lambda$ and $1 / \lambda$ are invariant by the action of $\mathbb{Z}^{2}$ on $\mathbb{R}^{2}$ and so induce on $\mathbb{T}^{2}$ two foliations which are invariant by $\varphi$. By suspension of these foliations on the 3 -manifold $\mathbb{T}_{\varphi}^{3}$ obtained by suspension of $\varphi$, we get two minimal foliations on $\mathbb{T}_{\varphi}^{3}$ which are called model foliations. Ghys and Sergiescu proved the following theorem:

Theorem 3.1 ([5]). Every codimension $1 C^{r}, r \geq 2$, transversely affine orientable foliation without compact leaf on $\mathbb{T}_{\varphi}^{3}$ is $C^{r-2}$-conjugated to a model foliation.

Now suppose that the fiber is a closed surface of genus $\geq 2$. According to Proposition 2.2, the monodromy $\varphi$ is pseudo-Anosov, we suppose in addition that the fiber is oriented. We denote $\omega^{+}, \omega^{-}$the characteristic 1 -forms of $\varphi$ and $\lambda$ the coefficient of dilation. Let us construct the model foliations on $V$.

\subsection{Elementary foliations on $\mathbb{D}^{2} \times \mathbb{S}^{1}$}

We will construct two foliations on $\mathbb{D}^{2} \times \mathbb{S}^{1}$ transverse to boundary $\partial\left(\mathbb{D}^{2} \times \mathbb{S}^{1}\right)$ which will serve as surgery of model foliations on the whole bundle.

\section{A foliation without singularity on $\mathbb{T}^{2}$}

On $\mathbb{S}^{1}=[0,2] / 0 \sim 2$, we consider the function $f$ defined by

$$
\begin{aligned}
f:[0,2] & \rightarrow \mathbb{R} \\
x & \mapsto f(x)=-\cos 2 \pi x .
\end{aligned}
$$


We have $f^{-1}(0)=\left\{\frac{1}{4}, \frac{3}{4}, \frac{5}{4}, \frac{7}{4}\right\}$, we consider on $\mathbb{T}^{2}$, the foliation $\mathcal{F}_{k}$ defined by the 1 -form $\omega_{k}=d f+k f d z$, where $(x, z)$ are the coordinates on $\mathbb{R}^{2}$ and $\mathbb{T}^{2}=\mathbb{R}^{2} / \mathbb{Z}^{2}$. The only compact leaves of $\mathcal{F}_{k}$ are $T \times \mathbb{S}^{1}$, where $T$ is a connected component of $f^{-1}(0)$. The compact leaves have the holonomy and the no compact leaves roll up to compact leaves. So $\mathcal{F}_{k}$ is a foliation with four Reeb plane components (see Figure 3); $\mathcal{F}_{k}$ is a one parameter family of transversely affine foliations with holonomy on $\mathbb{T}^{2}$.

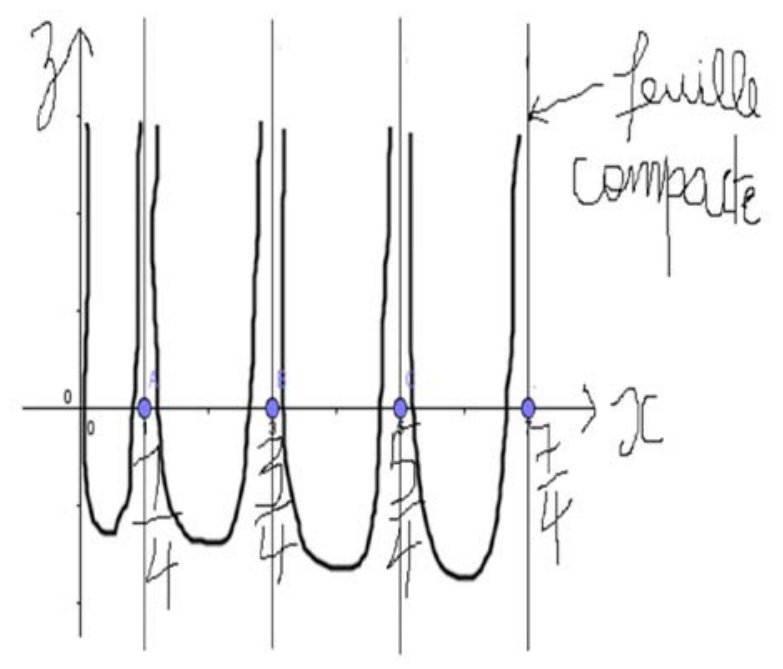

Figure 3. Nonsingular foliation on the torus (feuille compacte $=$ compact leaf).

\section{A family of regular foliations on $\mathbb{D}^{2} \times \mathbb{S}^{1}$}

Consider the 1 -form $\omega_{0}=x d x-y d y$ of saddle type on $\mathbb{R}^{2}$ and the 1-form $\widetilde{\Omega}_{\lambda}=\lambda^{z} \omega_{0}+d z, \lambda \in \mathbb{N}^{*}$ on $\mathbb{R}^{2} \times \mathbb{R}$. It is the nonsingular 1-form satisfying: $d \widetilde{\Omega}_{\lambda}=\widetilde{\alpha}_{\lambda} \wedge \widetilde{\Omega}_{\lambda}$, where $\widetilde{\alpha}_{\lambda}=(\log \lambda) d z$. Then $\widetilde{\Omega}_{\lambda}$ defines a transversely affine foliation on $\mathbb{R}^{2} \times \mathbb{R}$. We consider the function 


$$
\begin{aligned}
h_{\lambda}: \mathbb{R}^{2} \times \mathbb{R} & \rightarrow \mathbb{R}^{2} \times \mathbb{R} \\
(u, z) & \mapsto(\lambda u, z+1) .
\end{aligned}
$$

The quotient by the action generated by $h_{\lambda}$ gives a cyclic covering $\mathbb{R}^{2} \times \mathbb{R} \rightarrow \mathbb{R}^{2} \times \mathbb{S}^{1}$. The ordered pair $\left(\widetilde{\Omega}_{\lambda}, \tilde{\alpha}_{\lambda}\right)$ is invariant by this action and passes to the quotient in an ordered pair $\left(\Omega_{\lambda}, \alpha_{\lambda}\right)$ which defines a regular foliation on $\mathbb{R}^{2} \times \mathbb{S}^{1}$.

\subsection{The model foliations on $V$}

Now we can construct the model foliations on $V$ using the GhysThurston method sketched in many works one of which being [12]. The method consists of a suspension of the stable and unstable foliations followed by a desingularization. On $\sum \times \mathbb{R}$, we consider the 1 -form $\Omega^{+}=\lambda^{t} \omega^{+}+\psi d t$, where $\phi$ is $e^{\infty}$ with support in a canonical neighbourhood of singularities of $\omega^{+}$. The form $\Omega^{+}$is invariant by the action $(x, t) \sim(\varphi(x), t+1)$ and passes to the quotient $V=\sum \times \mathbb{R} / \sim$ in a minimal transversely affine and singular foliation $\mathcal{H}^{+}$which has a finite number of contact circles $\gamma_{1}, \ldots, \gamma_{n}$ with the fibration of $V$ over $\mathbb{S}^{1}$. According to the last constructions and the lemma below, each circle $\gamma_{i}$ has a tubular neighbourhood $V_{i}$ diffeomorphic to $\mathbb{D}^{2} \times \mathbb{S}^{1}$ foliated as in the figure below (Figure 2), such that $\partial V_{i}$ is transverse to $\mathcal{H}^{+}$and $\mathcal{H}_{\mid \partial V_{i}}^{+}$ is the foliation $\mathcal{F}_{k}$ on the torus $\mathbb{T}^{2}$ described above for $k=\log \lambda$. We define in the same way $\mathcal{H}^{-}$using $\omega^{-}$. 


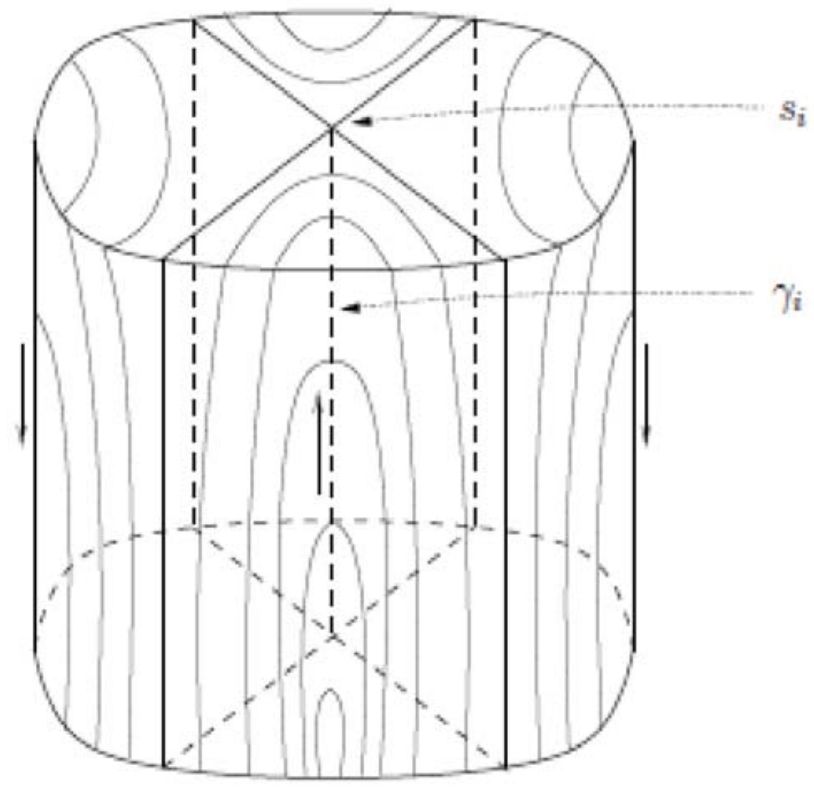

Figure 4. Tubular neighbourhood $V_{i}$.

Lemma 3.1. $\mathcal{H}^{+}$or $\mathcal{H}^{-}$is fixed as above.

(a) There exists a neighbourhood $M \subset V$ of $\{0\} \times \mathbb{S}^{1}$ diffeomorphic to $\mathbb{D}^{2} \times \mathbb{S}^{1}$ such that the boundary $\partial M$ of $M$ is transverse to $\mathcal{H}^{+}$or $\mathcal{H}^{-}$.

(b) For all $M$ satisfying (a), the trace of $\mathcal{H}^{+}$or $\mathcal{H}^{-}$on $\partial M$ is diffeomorphic to foliation $\mathcal{F}_{k}$ of $\mathbb{T}^{2}$ for $k=\log \lambda$, or $k=-\log \lambda$.

Proof. Let $\sigma_{1}, \sigma_{2}, \sigma_{3}$, and $\sigma_{4}$ be the separatrices of the saddle singularity 0 of $\Omega^{+}$. All the leaves $\sigma_{i} \times \mathbb{S}^{1}$ of $\mathcal{H}^{+}$have holonomy. We can take a tubular neighbourhood $M \subset V$ of $0 \times \mathbb{S}^{1}$ such that $\partial M \simeq \mathbb{T}^{2}$ is transverse to $\mathcal{H}^{+}$. The holonomy of the leaves $\sigma_{i} \times \mathbb{S}^{1}$ is determined by the linear holonomy $-\log \lambda d z$. Therefore $\mathcal{H}_{\mid \partial M}^{+}$is a foliation on torus $\mathbb{T}^{2}$ with four circular compact leaves having holonomy. The other leaves of 
$\mathcal{H}_{\mid \partial M}^{+}$are not compact and they spiral on the four compact leaves to give a foliation of $\mathbb{T}^{2}$ with four Reeb plane components and whose compact leaves have $-\log \lambda$ as coefficient of holonomy; $\mathcal{H}_{\mid \partial M}^{+}$is then a foliation of type $\mathcal{F}_{k}$ for $k=\log \lambda$.

We desingularize the foliations $\mathcal{H}^{+}$and $\mathcal{H}^{-}$as follows :

Let $S$ be the finite singular set common to $\omega^{+}$and $\omega^{-}$. We have constructed above two foliations on $\mathbb{D}^{2} \times \mathbb{S}^{1}$ which are used to complete $\mathcal{H}^{+}$and $\mathcal{H}^{-}$by surgery on whole 3 -manifold $V$. On $\left(\sum-S\right) \times \mathbb{R}$, the 1-forms $\lambda^{t} \omega^{+}+d t$ and $\lambda^{t} \omega^{+}-d t$ define two nonsingular foliations $\mathcal{V}_{1}^{+}$ and $\mathcal{V}_{2}^{+}$on $V-S^{\prime}$, with $S^{\prime}=U \gamma_{i}$, which have respectively $\chi(T \pi)$ and $-\chi(T \pi)$ as Euler classes. Each circle $\gamma_{i}$ of $S^{\prime}$ has a neighbourhood $W_{i}$ whose boundary is transverse to $\mathcal{V}_{i}(i=1,2)$. So we cut the $V_{i}$ and they are replaced by the $W_{i}$ foliated as in the figures below (Figures 5 and 6).

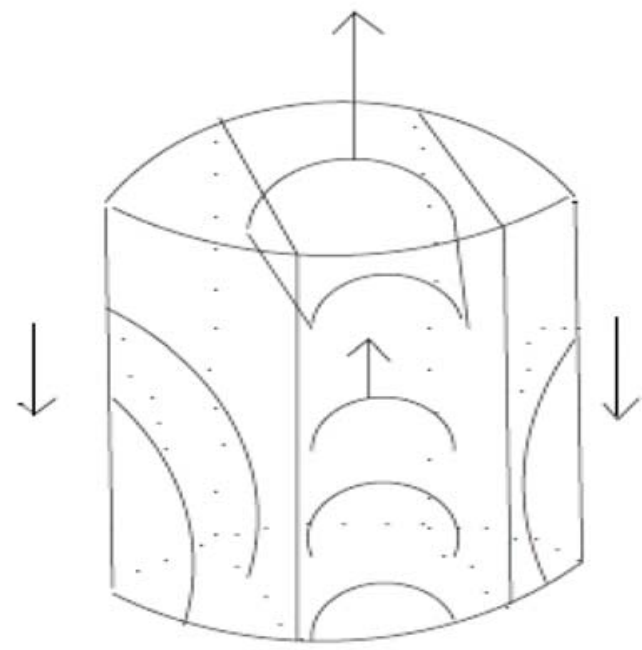

Figure 5. Tubular neighbourhood $\left(W_{i}, \mathcal{V}_{1}\right)$ 


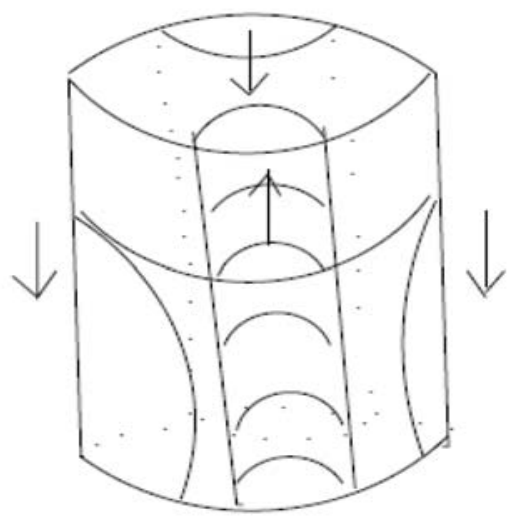

Figure 6. Tubular neighbourhood $\left(W_{i}, \mathcal{V}_{2}\right)$.

When the transverse orientation of $\mathcal{H}^{+} \mid \partial V_{i}$ is the same as the orientation of $\gamma_{i}$, we replace $V_{i}$ by $W_{i}$ with foliation $\mathcal{V}_{1}$, otherwise $V_{i}$ is replaced by $W_{i}$ with foliation $\mathcal{V}_{2}$. We obtain two regular foliations $\mathcal{F}_{+}^{1}$ and $\mathcal{F}_{+}^{2}$ transversely affine without compact leaf on $V$. Likewise, $\mathcal{H}^{-}$ gives two regular foliations $\mathcal{F}_{-}^{1}$ and $\mathcal{F}_{-}^{2}$. According to the formulas (1) and (2), the Euler number of $\mathcal{F}_{+}^{i}$ and $\mathcal{F}_{-}^{i}$ is an even integer $n$ such that $|n| \leq-\chi^{(\Sigma)}$.

\section{Deformations of Foliations and Classification}

The usual technique in studying foliations of 3-manifolds is to restrict them to their 2 dimensional submanifolds. But the foliations induced on those surfaces are not always simple. Indeed, they have singularities which are generically of Morse type (i.e., saddle or center). In some cases the singularities can be reduced.

Definition 4.1. Let $\mathcal{F}$ be a codimension 1 foliation on a hyperbolic bundle $\pi: V \rightarrow \mathbb{S}^{1}$. 
(a) We say that $\mathcal{F}$ is in general position with respect to the fibration $\pi$ if:

(i) There exists a finite family $\Gamma \subset V$ of embedded circles (said braid) such that $T_{u} \mathcal{F}=T_{u} \pi$ if and only if $u \in \Gamma$.

(ii) Every component $\gamma$ of $\Gamma$ has a tubular neighbourhood $V(\gamma)$ in which $\mathcal{F}$ is transverse to $\pi$ except at a finite number of points (restricted to any fiber) which are singularities of type saddle or center type.

(b) $\mathcal{F}$ is in improved general position if in (ii) we have only saddle singularities.

Theorem 4.1. Let $V$ be a closed 3-manifold and $\mathcal{F}$ a $C^{2}$ taut foliation on $V$. Let $\rho: \sum \rightarrow V$ be an embedding of a closed surface $\sum$ in $V$. We assume that $\rho_{*}: \pi_{1} \sum \rightarrow \pi_{1} V$ is injective. Then $\rho$ is isotopic to an embedding $\rho^{\prime}$ which is tangent to $\mathcal{F}$, or transverse to $\mathcal{F}$ except at a finite number of points which are saddle singularities (unrelated to each other) with an even number of separatrices for $\mathcal{F}$.

This theorem is proved by Roussarie in the case where the surface $\sum$ is torus $\mathbb{T}^{2}$ (see [17]). The same technique can be used for a surface of any genus. Here we show this theorem using a method of Riemannian geometry.

Proof. We know that according to Hass result [13] there is a Riemannian metric $g$ making all leaves of $\mathcal{F}$ minimal submanifolds. Every fiber $\sum$ is incompressible and so isotopic to a minimal orientable and closed surface.

Applying Schoen-Yao result [18] we can say that $\rho$ is isotopic to an embedding $\rho^{\prime}$ which is minimal relatively to $g$ and we use the same result on the intersections of minimal surfaces according to which the trace of a minimal foliation on a minimal surface which is not a leaf is a 
singular foliation whose saddle singularities have an even number of separatrices. Then, by small isotopies, we can explode the $2 n$-branch saddle singularities into 4-branch isolated saddle singularities.

Proposition 4.1. Let $V$ be a closed 3-manifold bundle over circle $\mathbb{S}^{1}$ and $\mathcal{F}$ a $C^{2}$ taut foliation on $V$. If $\mathcal{F}$ has no compact leaf, then every fiber of $V$ is isotopic to a closed surface in optimal position (i.e., transverse to $\mathcal{F}$ except at a finite number of points which are saddles with 4 separatrices).

Proof. Indeed, the exact sequence of homotopy associated to the fibration shows that the canonical injection of each fiber of $V$ is injective at the level of the fundamental groups. Then we apply the theorem above to obtain the points of contact which are saddles with an even numbers of separatrices, by sequence of small isotopies, we can explode all saddles into isolated saddles with 4 separatrices.

Applying the desingularization above and using the Matsumoto theorem [12], we prove the following theorem:

Theorem 4.2. Let $V$ be a closed 3-manifold bundle over circle $\mathbb{S}^{1}$ with the pseudo-Anosov monodromy. If the fibers of $V$ are of genus $g$, then there are at least $4 \mathrm{~g}-2$ isotopy classes of transversely affine and taut foliations on $V$.

Proof. Indeed, for all $|k| \leq-\chi\left(\sum\right) / 2$, there is a taut transversely affine foliation $\mathcal{F}_{k}$ whose Euler number is $2 k$, since it is sufficient to suppose that $\Omega^{+}$and $\Omega^{-}$have $\left(k-\chi\left(\sum\right)\right)$ positive singularities and $\left(-k-\chi\left(\sum\right)\right)$ negative singularities and to apply the last construction (see the model construction).

In order to study the effects of a perturbation of a codimension 1 foliation on the 3-manifolds, we introduce the notion of the symplectic fillability of plane fields. 
Definition 4.2. Let $\xi_{1}$ and $\xi_{2}$ be two plane fields on $V$, we shall say that $\xi_{1}$ is a perturbation of $\xi_{2}$ if for all $x \in V$, the tangent planes to $\xi_{1}$ and $\xi_{2}$ are $\boldsymbol{e}^{0}$-close.

The following theorem makes it's possible to pass from a foliation to a contact structure.

Theorem 4.3 ([19]). Every $e^{2}$ codimension 1 foliation on $V$, different from a foliation of $\mathbb{S}^{2} \times \mathbb{S}^{1}$ in spheres, can be perturbated into a contact structure.

Definition 4.3. We suppose that $(V, \xi)$ is a boundary of a symplectic 4-manifold $(W, \omega) .(W, \omega)$ is called a symplectic filling of a contact structure or a foliation $(V, \xi)$ if the restriction of the 2 -form $\omega$ to $\xi$ has no singularity. In that case we say that $\xi$ is symplectically fillable.

If $V$ is a connected component of the boundary of $W$, then $\xi$ is called symplectically semi-fillable.

For example, it's well known [6] that if $V$ is an oriented closed 3-manifold which is $\sum$-bundle over circle $\mathbb{S}^{1}$ and $\xi$ is a plane field which is tangent to fibers then $(V, \xi)$ is, up to finite covering, symplectically fillable.

Theorem 4.4 ([19]). (i) Symplectically semi-fillable contact structures on $V$ are tight (i.e., not overtwisted).

(ii) Taut foliations on V are symplectically semi-fillable.

We always consider that $V$ is a hyperbolic and a bundle over $\mathbb{S}^{1}$ with fibration $\pi$. The following theorem shows that all taut foliations on $V$ do not always result from $\pi$ by perturbation.

Theorem 4.5. There exist hyperbolic closed 3-manifolds bundles over circle $\mathbb{S}^{1}$ with the fibration $\pi$ and atoroidals having taut foliations which do not result from perturbations of fibration $\pi$. 
The proof of this theorem is based on the following lemmas: Let $V$ be any closed atoroidal 3-manifold bundle over $\mathbb{S}^{1}$, and $\sum$ be the fiber of $V$, then $\sum$ is a closed surface with genus $g>1$. Denote $\varphi: \sum \rightarrow \sum$ the monodromy map of $\pi$, the manifold $V$ is diffeomorphic to the suspension of $\varphi$, i.e., $V=\sum \times[0,1] /(x, 0) \sim(\varphi(x), 1)$.

Lemma 4.1. The diffeomorphism $\varphi$ is isotopic to a pseudo-Anosov one.

Proof. We know from [2] that $\varphi$ is isotopic to a diffeomorphism which is periodic or irreducible or pseudo-Anosov. The manifold $V$ is sufficiently large since the fundamental groups of the fibers are injected into that of $V$. The manifold obtained by cutting $V$ along the fiber $\sum$ is diffeomorphic to $\sum \times[0,1]$. If $\varphi$ is of finite order, the foliation by interval of $\sum \times[0,1]$ gives, by identification of components of the boundary by $\varphi$, a manifold bundle in circles, which is a contraduction because $V$ is irreducible, atoroidal and contains embedded surfaces whose the fundamental groups are injected in that of $V$. If $\varphi$ est irreducible, then $\varphi$ makes invariant a finite collection of simple closed embedded curves $\Gamma$. After suspension of $\varphi$ those curves give the embedded incompressible tori, which is a contraduction since $V$ is atoroidal.

Lemma 4.2. Let $\xi_{1}$ and $\xi_{2}$ be two foliations or contact structures on $V$. If $\xi_{1}$ is a perturbation of $\xi_{2}$, then the Euler classes e $\left(\xi_{1}\right)$ of $\xi_{1}$ and $e\left(\xi_{2}\right)$ of $\xi_{2}$ are equal.

Proof. The manifold $V$ being oriented, as well as the plane fields $\xi_{1}$ and $\xi_{2}$, if the perturbation is sufficiently small, then $\xi_{1}$ and $\xi_{2}$ remain transverse to the same normal bundle. Therefore, they are isomorphic and have the same Euler class. 
Proof (of Theorem 4.5). Let $V$ be a closed atoroidal 3-manifold bundle over circle $\mathbb{S}^{1}$ with a fibration $\pi$. According to Lemma 4.1, the monodromy $\varphi$ is isotopic to a pseudo-Anosov diffeomorphism which we can also denote $\varphi$. Let $g$ be the genus of the fibers $\sum_{g}$ of $\pi$. Then there exist two transverse singular measured foliations $\mathcal{F}^{s}$ and $\mathcal{F}^{u}$ whose singularities are saddles with $2 p$ separatrices and a real $\lambda>1$ such that

$$
\varphi_{*}\left(\mathcal{F}^{s}, \mu^{s}\right)=\left(\mathcal{F}^{s}, \lambda^{-1} \mu^{s}\right)
$$

and

$$
\varphi_{*}\left(\mathcal{F}^{u}, \mu^{u}\right)=\left(\mathcal{F}^{u}, \lambda \mu^{u}\right) .
$$

Denote $S=\left\{s_{1}, s_{2}, \ldots, s_{2 g-2}\right\}$ the common singular set of foliations $\mathcal{F}^{s}$ and $\mathcal{F}^{u}$. On $\left(\sum_{g}-S\right) \times \mathbb{R}$, we consider the foliation defined by the 1 -form $\Omega_{\sigma}=\lambda^{\varepsilon(\sigma) t} \omega^{\sigma}$, where $\omega^{\sigma}$ is a closed 1-form defining $\mathcal{F}^{\sigma}$ with $\varepsilon(\sigma)=1$ if $\sigma=s$ and $\varepsilon(\sigma)=-1$ if $\sigma=u$. $\Omega_{\sigma}$ defines on $V$ a singular foliation $\tilde{\mathcal{F}}^{\sigma}$ whose singular set is $(S \times \mathbb{R}) /(x, t) \sim(\varphi(t), t+1)$.

We can extend $\tilde{\mathcal{F}}^{\sigma}$ into a nonsingular foliation $\mathcal{F}^{\sigma}$ on $V$, since $s_{i}$ are 4 -saddles (with an even number of separatrices).

As the $s_{i}$ are 4-saddles, formulas (1) and (2) introduced in second paragraph become :

$\left\{\begin{array}{l}\text { number of }\left(s_{i} \text { negative }\right)-\text { number of }\left(s_{i} \text { positive }\right)=e(T \mathcal{F}) \cdot\left[\sum\right] ; \\ \text { number of }\left(s_{i} \text { positive }\right)+\text { number of }\left(s_{i} \text { negative }\right)=-\chi\left(\sum\right) .\end{array}\right.$

Let $n>0$ be an integer such that $-\chi\left(\sum\right)=2 n$ and $\tilde{\mathcal{F}}^{\sigma}$ has $(n-1) /$ $2 \chi\left(\sum\right)$ negative singularities and $(-n-1) / 2 \chi\left(\sum\right)$ positive singularities. 
Then $e(T \mathcal{F}) \cdot\left[\sum\right]$ is different from $-\chi\left(\sum\right)$. So the Euler class $e\left(\mathcal{F}^{\sigma}\right)$ of $\mathcal{F}$ is different from $e(\pi)$. So from Lemma $4.2, \mathcal{F}^{\sigma}$ is not a perturbation of fibration $\pi$.

However for such manifolds, the fibration and its perturbations are the only taut foliations in the Euler class of fibration. Precisely we have:

Theorem 4.6. Let $V$ be an atoroidal closed 3-manifold $\sum$-bundle over circle $\mathbb{S}^{1}$, with a fibration $\pi$. Let $\mathcal{F}$ be a transversely orientable foliation of codimension 1 on $V$. Then $\mathcal{F}$ is a taut foliation having the same Euler class as the fibration $\pi$ if and only if $\mathcal{F}$ is a perturbation of $\pi$.

Proof. $\Leftarrow$ The proof in one sense is obvious.

Indeed the fibration $\pi$ being taut, there is a closed curve $\gamma$ transverse to all fibers. If $\mathcal{F}$ is a perturbation sufficiently small of $\pi, \gamma$ remain transverse to all the leaves of $\mathcal{F}$ since the transversality is an open propriety. So $\mathcal{F}$ is taut; furthermore $\mathcal{F}$ has the same Euler class as $\pi$ from Lemma 4.2 .

$\Rightarrow$ Inversely, suppose that $\mathcal{F}$ is taut and has the same Euler class as fibration $\pi$. We use the following lemma:

Lemma 4.3. The foliation $\mathcal{F}$ and the fibration $\pi$ are perturbed respectively into two taut and isotopic contact structures $\xi_{1}$ and $\xi_{2}$.

Proof. According to Theorem 4.3, $\pi$ is perturbed into a contact structure $\xi_{1}$. As $\pi$ is taut, it is symplectically semi-fillable. As symplectic semi-fillableness is $C^{0}$-open propriety, $\xi_{1}$ is symplectically semi-fillable. But symplectically semi-fillable contact structures are tight, so $\xi_{1}$ is tight. Likewise, $\mathcal{F}$ is perturbed into a tight contact structure $\xi_{2}$. As $\mathcal{F}$ and $\pi$ have the same Euler class, so do $\xi_{1}$ and $\xi_{2}$. From Lemma 4.1, the manifold $V$ is diffeomorphic to a suspension of a diffeomorphism 
isotopic to a pseudo-Anosov $\varphi$, which is isotopic to a monodromy of $V$. Therefore from Kazez result, $\xi_{1}$ is isotopic to $\xi_{2}[15]$.

Thus the foliation $\mathcal{F}$ is a perturbation of the fibration $\pi$.

The following theorem gives a classification of taut foliations in improved general position with respect to the fibration on a toroidal 3-manifolds bundles over circle $\mathbb{S}^{1}$.

Theorem 4.7. Let $\mathcal{F}$ be a codimension 1 taut foliation on a closed atoroidal 3-manifold which is a hyperbolic bundle $\pi: V \rightarrow \mathbb{S}^{1}$ over $\mathbb{S}^{1}$ with monodromy $\varphi: \sum \rightarrow \sum$, where $\sum$ is a closed oriented surface of genus $g>1$ and having the same Euler class as the fibration. Let $\tilde{\mathcal{F}}$ be a foliation induced by $\mathcal{F}$ on $\sum \times[0,1]$ obtained by cutting $V$ along a fiber $\sum$. If the foliation $\mathcal{F}$ is in improved general position with respect to fibration $\pi$, then $\tilde{\mathcal{F}}$ is isotopic to a product foliation $\widetilde{\mathcal{F}}_{0} \times[0,1]$, where $\widetilde{\mathcal{F}}_{0}$ is isotopic to a characteristic foliation of $\varphi$.

Proof. For the proof of this theorem, we suppose that $\mathcal{F}$ has no compact leaf. The foliation $\mathcal{F}$ being in improved general position with respect to fibration $\pi$, let $\sum$ be a fiber in optimal position and $\tilde{\mathcal{F}}$ the restriction of $\mathcal{F}$ to $\sum \times[0,1]$ obtained by cutting $V$ along $\sum$. Denote $\mathcal{F}_{0}$ and $\mathcal{F}_{1}$ the foliations induced by $\mathcal{F}$ on the boundaries $\sum \times\{0\}$ and $\sum \times\{1\}$.

The foliation $\mathcal{F}$ being taut and having the same Euler class as the fibration, it is a perturbation of fibration $\pi$ and so it is defined by a closed 1-form; and the foliations $\mathcal{F}_{0}$ and $\mathcal{F}_{1}$ induced respectively on $\sum \times\{0\}$ and $\sum \times\{1\}$ are defined by the singular closed 1-forms which we 
denote respectively $\omega_{0}$ and $\omega_{1}$, we have $\omega_{1}=\varphi_{*} \omega_{0}$. We can suppose that $\omega_{0}$ and $\omega_{1}$ are cohomologous. So we have $\omega_{1}-\omega_{0}=d f$, where $f$ is a real function on $V$. As $\mathcal{F}$ is a perturbation from the fibration $\pi$, there is a vector field $X$ transverse to both $\mathcal{F}$ and $\pi$, so $X$ is transverse to $\mathcal{F}_{0}$ and $\mathcal{F}_{1}$.

On $\sum \times[0,1]$ we define the 1 -form

$$
\Omega_{(x, t)}=t \omega_{0}+(1-t) \omega_{1}+f(x) d t
$$

for all $(x, t) \in \sum \times[0,1]$. The form $\Omega$ is nonsingular and closed, it defines a foliation $\mathcal{H}$, transverse to all fibers $\sum \times\{t\}$ except at a finite number of points since $\Omega(X)>0$, which induced $\mathcal{F}_{0}$ and $\mathcal{F}_{1}$. Therefore $\mathcal{F}_{0}$ and $\mathcal{F}_{1}$ are isotopic according to the following lemma:

Lemma 4.4. Let $\mathcal{F}_{0}$ and $\mathcal{F}_{1}$ be two singular foliations whose singularities are saddles with an even number of separatrices on a closed surface $S$. If there exists a foliation $\mathcal{H}$ on $S \times[0,1]$ inducing $\mathcal{F}_{0}$ and $\mathcal{F}_{1}$ and transverse to fibers $S \times\{t\}$, then $\mathcal{F}_{0}$ and $\mathcal{F}_{1}$ are isotopic.

Proof. By transversality the natural projection $p: S \times$ $[0,1] \rightarrow[0,1]$ defines a submersion $p_{*}: T_{(x, t)} \mathcal{H} \rightarrow T_{t}[0,1]$. We can choose the section $s$ of tangent bundle of $\mathcal{H}$ which is horizontal (i.e., $p_{*} s$ $=\frac{\partial}{\partial t}$ ). And as well as $T \mathcal{H}$ is locally trivial, we construct such a field in every distinguished open set and we make a gluing with a smooth partition of unity. Denote $\varphi_{t}(x, 0)$ the flow of $s$ passing through $(x, 0)$. As $S$ is compact, the flow $\varphi_{t}(x, 0)$ of $s$ is defined for all $t \in[0,1]$ and all $x \in S$. This flow lies entirely in the leaf of $\mathcal{H}$ through $(x, 0)$ and at each time $t \in[0,1]$, meets the fiber $S \times\{t\}$, so $\varphi_{t}$ carries $\mathcal{F}_{0}=\mathcal{H}_{0}$ onto $\mathcal{H}_{t}$. We can suppose that the separatrices of each singularity of $\mathcal{F}_{0}$ are sent 
to the separatrices of singularities of $\mathcal{H}_{t}$. The desired isotopy can be defined by $\psi(x, t)=\pi \circ \varphi_{t}(x, 0)$, where $\pi: S \times[0,1] \rightarrow S$.

The rest of the proof of Theorem 4.7. $\forall t \in[0,1], \tilde{\mathcal{F}}_{\mid \sum \times\{t\}}$ is isotopic to $\mathcal{F}_{0}=\tilde{\mathcal{F}}_{\mid \sum \times\{0\}}$. The foliation $\tilde{\mathcal{F}}$ is so isotopic to the product foliation $\widetilde{\mathcal{F}}_{0} \times[0,1]$ on $\sum \times[0,1]$. From the previous results, the isotopy class of $\mathcal{F}_{0}$ is an invariant of pseudo-Anosov $\varphi$ isotopic to the monodromy of $V\left(\right.$ i.e., $\left.\varphi_{*}\left(\mathcal{F}_{0}\right)=\mathcal{F}_{1}\right)$ and as $\mathcal{F}$ has no compact leaf $\mathcal{F}_{0}$ is isotopic to a foliation of a pseudo-Anosov.

\section{References}

[1] A. Candel and L. Conlon, Foliations I, Graduate Studies in Mathematics, American Mathematical Society 13.

[2] A. Fathi, F. Laudenbach and V. Poenaru, Travaux de Thurston sur les surfaces, S.M.F. Astérisque (1979), 66-67.

[3] B. Seke, Sur les structures transversalement affines des feuilletages de codimension 1, Ann. Inst. Fourier 30 (1980), 1-29.

[4] D. Tischler, On fibering certain manifold over the circle, Topology 9 (1970), 153-154.

[5] E. Ghys and V. Sergiescu, Stabilité et Conjugaison différentiable pour certains feuilletages, Topology 19 (1980).

[6] E. Giroux, Topologie de contact en dimension 3, Séminaire N. Bourbaki 760 (1992-1993), 7-33.

[7] F. Laudenbach and S. Blank, Sur l'isotopie des formes fermées en dimension 3, Ivent. Math. 54 (1979), 103-177.

[8] G. Levitt, Pantalons et feuilletages des surfaces, Topology 1 (1982), 9-33.

[9] H. Dathe, Sur les feuilletages tendus transversalement affines des 3-variétés fibrées sur $\mathbb{S}^{1}$, African Diaspora Journal of Mathematics 9(2) (2010), 17-33.

[10] H. Dathe and A. Saidou, Feuilletages sans feuille compacte sur les fibrés, en surfaces sur le cercle, African Diaspora Journal of Mathematics 11(2) (2011), 78-89.

[11] H. Dathe and C. Tarquini, Sur les feuilletages des variétés fibrées, Annales Mathématiques Blaise Pascal 15(2) (2008), 211-232.

[12] H. Nakayama, Transversely affine foliations on some surfaces bundles over $\mathbb{S}^{1}$ of pseudo-Anosov type, Ann. Inst. Fourier 41 (1991). 
[13] J. Hass, Minimal surfaces in manofolds, Comment Math. Helv 61 (1986), 1-32.

[14] J. P. Otal, Le théorème hyperbolisation pour les variétés fibrées de dimension 3, S.M.F. Astérisque 235 (1996).

[15] K. Honda, W. Kazez and G. Matic, Tight contact srtuctures on fibered hyperbolic 3-manifolds, J. Differential Geometry 64 (2003), 305-358.

[16] R. Bieri, D. Neumann and R. Strebel, A geometric invariant of discrete groups, Invent. Math. 90 (1987), 451-477.

[17] R. Roussarie, Plongements dans les variétés feuilletées et classification de feuilletages sans holonomie, Publ. Math. IHES 43 (1974), 101-141.

[18] R. Schoen and S. Yao, Existence of incompressible minimal surfaces and the topology of three dimensional manifolds with non-negative scalar curvature, Ann. Math. 110 (1979), 127-142.

[19] Y. M. Eliasberg and W. P. Thurston, Confoliations, University Lecture Series, Volume 13, A.M.S.

[20] W. Thurston, Norm on the homology of 3-manifolds, Memoirs of the A.M.S. 339 (1986), 99-130. 